\title{
CYP2D6 genetic polymorphisms and phenotypes in different ethnicities of Malaysian breast cancer patients
}

\begin{abstract}
ABSTRAK
The cytochrome P450, family 2, subfamily D, polypeptide 6 (CYP2D6) is an enzyme that is predominantly involved in the metabolism of tamoxifen. Genetic polymorphisms of the CYP2D6 gene may contribute to inter-individual variability in tamoxifen metabolism, which leads to the differences in clinical response to tamoxifen among breast cancer patients. In Malaysia, the knowledge on CYP2D6 genetic polymorphisms as well as metabolizer status in Malaysian breast cancer patients remains unknown. Hence, this study aimed to comprehensively identify CYP2D6 genetic polymorphisms among 80 Malaysian breast cancer patients. The genetic polymorphisms of all the 9 exons of CYP2D6 gene were identified using high-resolution melting analysis and confirmed by DNA sequencing. Seven CYP2D6 alleles consisting of CYP2D6*1, CYP2D6*2, CYP2D6*4, CYP2D6*10, CYP2D6*39, CYP2D6*49, and CYP2D6*75 were identified in this study. Among these alleles, CYP2D6*10 is the most common allele in both Malaysian Malay (54.8\%) and Chinese $(71.4 \%)$ breast cancer patients, whereas CYP2D6*4 in Malaysian Indian $(28.6 \%)$ breast cancer patients. In relation to CYP2D6 genotype, CYP2D6*10/*10 is more frequently observed in both Malaysian Malay (28.9\%) and Chinese $(57.1 \%)$ breast cancer patients, whereas CYP2D6*4/*10 is more frequently observed in Malaysian Indian (42.8\%) breast cancer patients. In terms of CYP2D6 phenotype, $61.5 \%$ of Malaysian Malay breast cancer patients are predicted as extensive metabolizers in which they are most likely to respond well to tamoxifen therapy. However, $57.1 \%$ of Chinese as well as Indian breast cancer patients are predicted as intermediate metabolizers and they are less likely to gain optimal benefit from the tamoxifen therapy. This is the first report of CYP2D6 genetic polymorphisms and phenotypes in Malaysian breast cancer patients for different ethnicities. These data may aid clinicians in selecting an optimal drug therapy for Malaysian breast cancer patients, hence improve the clinical outcome of the patients.
\end{abstract}

Keyword: CYP2D6; Malaysian breast cancer patients; genetic polymorphisms; tamoxifen 